\title{
Current Status of Brachytherapy for Prostate Cancer
}

\author{
Dong Soo Park \\ Department of Urology, Bundang CHA Hospital, CHA University College of Medicine, Seongnam, Korea
}

Brachytherapy was developed to treat prostate cancer 50 years ago. Current advanced techniques using transrectal ultrasonography were established 25 years ago. Transrectal ultrasound (TRUS) has enabled the prostate to be viewed with improved resolution with the use of modern ultrasound machines. Moreover, the development of software that can provide images captured in real time has improved treatment outcomes. Other new radiologic imaging technologies or a combination of magnetic resonance and TRUS could be applied to brachytherapy in the future. The therapeutic value of brachytherapy for early-stage prostate cancer is comparable to that of radical prostatectomy in long-term follow-up. Nevertheless, widespread application of brachytherapy cannot be achieved for several reasons. The treatment outcome of brachytherapy varies according to the skill of the operator and differences in patient selection. Currently, only three radioactive isotopes are available for use in low dose rate prostate brachytherapy: I-125, Pd-103, and Cs-131; therefore, more isotopes should be developed. High dose rate brachytherapy using Ir-192 combined with external beam radiation, which is needed to verify the long-term effects, has been widely applied in high-risk patient groups. Recently, tumor-selective therapy or focal therapy using brachytherapy, which is not possible by surgical extraction, has been developed to maintain the quality of life in selected cases. However, this new application for prostate cancer treatment should be performed cautiously because we do not know the oncological outcome, and it would be an interim treatment method. This technique might evolve into a hybrid of whole-gland treatment and focal therapy.

Key Words: Brachytherapy; Neoplasms; Prostate

This is an Open Access article distributed under the terms of the Creative Commons Attribution Non-Commercial License (http://creativecommons.org/licenses/by-nc/3.0) which permits unrestricted non-commercial use, distribution, and reproduction in any medium, provided the original work is properly cited.

\author{
Article History: \\ received 10 September, 2012 \\ accepted 28 September, 2012
}

Corresponding Author:

Dong Soo Park

Department of Urology, Bundang CHA Hospital, CHA University College of

Medicine, 59 Yatap-ro, Bundang-gu,

Seongnam 463-712, Korea

TEL: +82-31-780-5352

FAX: $+82-31-780-5323$

E-mail: dsparkmd@cha.ac.kr

\section{INTRODUCTION}

Radioisotope implantation for prostate cancer was developed approximately 50 years ago by Whitmore Jr. [1], who introduced the open retropubic approach to implant I-125. This initial technique failed to achieve precise seed placement and resulted in poor treatment quality.

With the advent of transrectal ultrasound (TRUS), a transperineal implant approach with the use of a template guidance system was developed in brachytherapy for prostate cancer approximately 25 years ago. Computer software for a treatment-planning system was developed initially as a preplanning technique, and eventually the real-time planning system was added. The widespread appli- cation of prostate-specific antigen (PSA) testing has led to a profound downward staging migration of prostate cancer [2]. Therefore, the vast majority of patients with newly diagnosed prostate cancer exhibit clinically localized disease. This clinical phenomenon can increase the incidence of the local treatments, e.g., radical prostatectomy and brachytherapy.

New radioisotopes such as $\mathrm{Pd}-103$ and $\mathrm{Cs}-131$ have been applied in low dose rate (LDR) prostate brachytherapy. High dose rate (HDR) brachytherapy using Ir-192 has been used with external beam radiotherapy (EBRT) in the treatment of high-risk prostate cancer.

In terms of effectiveness and morbidity, the treatment result of the current brachytherapy technique is com- 
parable with that of radical prostatectomy and EBRT. A comparison of the recurrence rates or survival outcomes of radical prostatectomy and brachytherapy has shown inconsistent biochemical recurrence and survival rates in different studies.

A recent limited trial of partial prostate gland treatment using brachytherapy has been applied for early-stage indolent prostate cancer. This treatment strategy will be developed and modified.

\section{LDR BRACHYTHERAPY}

\section{Indications and contraindications}

The best candidates for LDR brachytherapy are low-risk prostate cancer patients. The criteria for low-risk groups are a PSA level $<10 \mathrm{ng} / \mathrm{ml}$ and Gleason scores $\leq 6$. The next ideal candidates for LDR brachytherapy are intermediate-risk prostate cancer patients with organ-confined disease. This group includes patients with a Gleason score of 7 with either $3+4$ or $4+3$. A Gleason grade sum of $3+4$ is more appropriate than a sum of $4+3$ in the application of LDR brachytherapy alone. High-risk prostate cancer and a Gleason score of $7(4+3)$ are generally required for the combined treatment of EBRT and LDR brachytherapy. However, Gleason score 8 prostate cancer can be treated with high-dose LDR brachytherapy monotherapy [3].

The prostate gland size should preferably be $<50$ to 60 $\mathrm{ml}$ because a large prostate frequently overlaps with the pubic arch, which interferes with needle placement. Additionally, large prostates require more seeds to achieve the prescribed dose, which results in an increased risk of urinary morbidity. Patients with large prostates should be prescribed a combination of androgen blockade with luteinizing hormone releasing hormone analogue and anti-androgen to reduce the prostate size before implantation.

Patients who have a history of transurethral resection of the prostate should be treated with caution because of the increased risk for incontinence and superficial urethral necrosis. Patients with preexisting significant obstructive urinary symptoms are not ideal candidates for LDR brachytherapy.

\section{Planning}

Preplanned technique: A plan is generated from the TRUS imaging that is obtained several days before isotope implantation. A preplanned prescription dose of radioisotope is delivered to the prostate through the needles that are placed under ultrasound guidance. Radioactive seeds are deposited through the needles with an applicator or with preloaded seeds on a strand containing the preplanned number of seeds, which is accomplished by using a needle obturator and withdrawing the needle. This technique causes the seeds to be distributed too homogeneously throughout the prostate, which increases the central dose; therefore, modified needle placement and seed-loading is required.
Intraoperative real-time treatment planning: Intraoperative planning results in excellent clinical outcomes. An advantage of the preplanned technique is that the prostate is measured in the unanesthetized state, which can be changed during anesthesia and the procedure. Multiple needle placements cause distortion and swelling of the prostate gland, which leads to imprecise implantation. A real-time treatment planning technique using transrectal ultrasonography can obviate the preplanning and can be used to determine the prostate volume and shape, which are needed to calculate the radiation dose prescription for the target. The preplanning dose to the prostate was based on the Anderson I-125 nomogram. Currently, many modified techniques that reduce the periurethral prescription dose are applied [4]. However, this dose modification has a risk of failing to eradicate the possible transitional zone cancer. Intraoperative planning has been used with various software programs, including the I-plant TPS [5] conformal optimization system [6].

\section{Isotopes}

Radioisotopes I (iodine)-125, Pd (palladium)-103, and Cs (cesium)-131 have LDRs and low energy. I-125 is commonly used in LDR brachytherapy with a radiation dose of $145 \mathrm{~Gy}, 144 \mathrm{~Gy}$, or more. The prescribed dose of $145 \mathrm{~Gy}$ was suggested by the American Association of Physicists in Medicine Task Group 64 [7] and the European Society for Therapeutic Radiology and Oncology/European Association of Urology/European Organisation for Research and Treatment of Cancer recommendations on prostate brachytherapy [8]. The prescribed dose of 144 Gy was developed by Memorial Sloan Kettering Cancer Center.

Pd-103 may be appropriate for rapidly proliferating tumors because of its radiobiologic characteristics. The usual prescription radiation dose of $\mathrm{Pd}-103$ is 115 to $125 \mathrm{~Gy}$. Cs-131 was approved in 2003 by the Food and Drug Administration for use in brachytherapy for prostate cancer. The recommended prescription dose of Cs-131 is 115 Gy.

The half-lives of I-125, Pd-103, and Cs-131 are 59.4 days, 17 days, and 9.7 days, respectively. The mean photon energy levels emitted by I-125, Pd-103, and Cs-131 are 21 $\mathrm{KeV}, 27 \mathrm{KeV}$, and $29 \mathrm{KeV}$, respectively.

When the photon energy is stronger, fewer radioactive seeds are required. Hence, the initial dose rates of I-125, Pd-103, and Cs-131 are 7 cGy, 18 cGy, and 20 cGy, respectively, per hour. Shorter half-lives and stronger radioisotope energy used for prostate cancer could reduce the risk of adverse reactions (Table 1).

\section{Procedure}

Prostate sizes and shapes are different, and irregularities of the prostate are commonly observed. Attention should be given during the procedure because the implanted radioactive seeds cannot be modified after placement.

The steps of LDR brachytherapy incorporate anesthesia, positioning, planning, implantation, cystoscopic examina- 
TABLE 1. Radionuclides for low dose rate prostate brachytherapy

\begin{tabular}{lccccc}
\hline & & \multicolumn{2}{c}{ Radiation prescription dose (gray) } & & \multicolumn{2}{c}{$\begin{array}{c}\text { Initial dose rate } \\
\text { (cGy/h) }\end{array}$} \\
\cline { 3 - 5 } Radioisotope & Year applied & Monotherapy & $\begin{array}{c}\text { Combination with } \\
\text { EBRT }\end{array}$ & Half-life (day) & \\
\hline I-125 & 1965 & $145-144$ & $110+45-50$ & 59.4 & 7 \\
Pd-103 & 1986 & $125-115$ & $90+45-50$ & 17 & 18 \\
Cs-131 & 2003 & 115 & $90+45-50$ & 9.7 & 20 \\
\hline
\end{tabular}

EBRT, external beam radiotherapy.
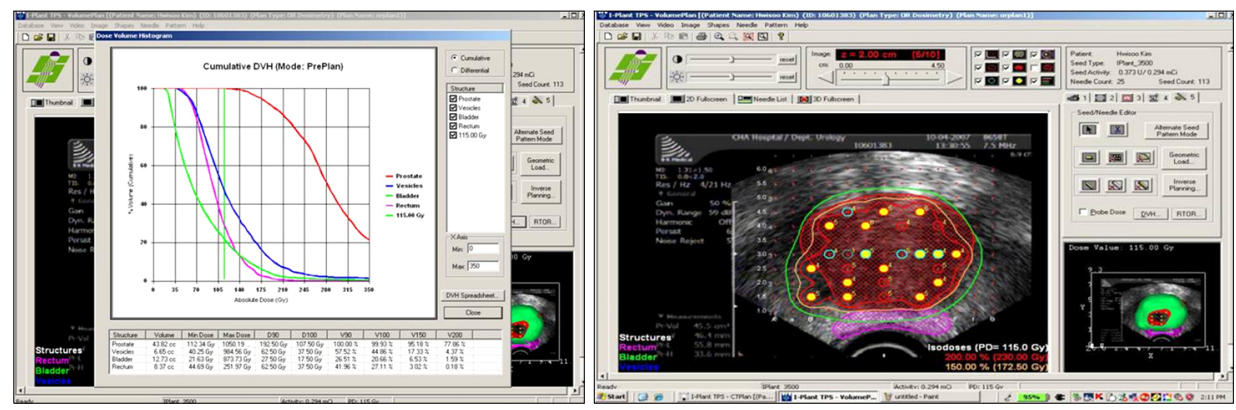

FiG. 1. Computer software for the intraoperative real-time dosimetry system.

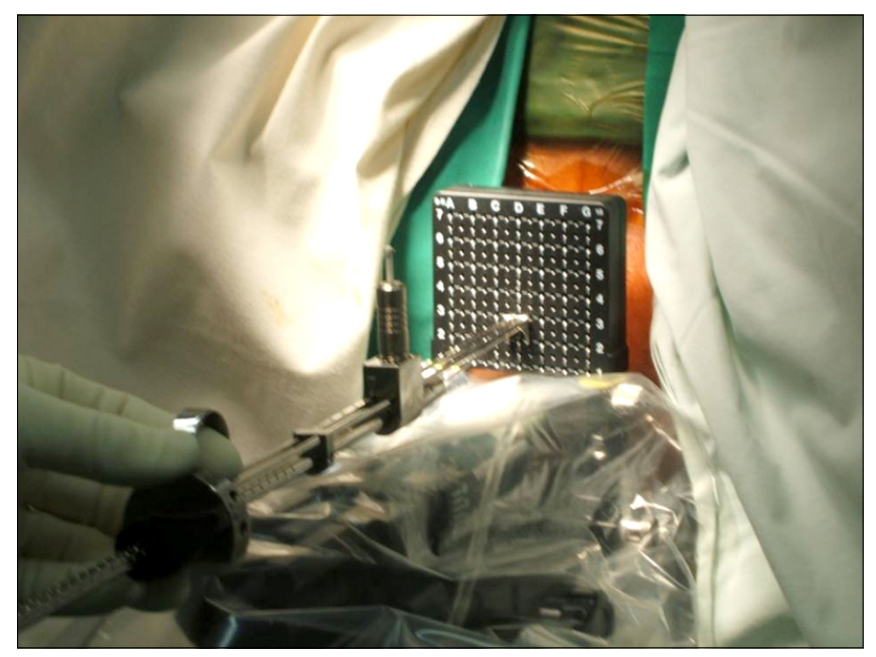

FiG. 2. Implantation is done through the perineum by using template guidance.

tion, seed count checking, and postimplant evaluation. General endotracheal or spinal anesthesia can be applied. Epidural anesthesia is not recommended because immobilization during the procedure is crucial.

A high and mildly extended lithotomy position is required. After the TRUS image capture of the prostate, the appropriate distribution of sources is determined by a dose-optimization treatment-planning computer software program (Fig. 1). The implantation is performed through the perineum (Fig. 2). The median number of needles used is approximately 20 to 35 . Cystoscopy is the final step to evaluate the urethra, bladder pathology, and misplaced seeds.

Normally, 1 month after the implantation to allow for

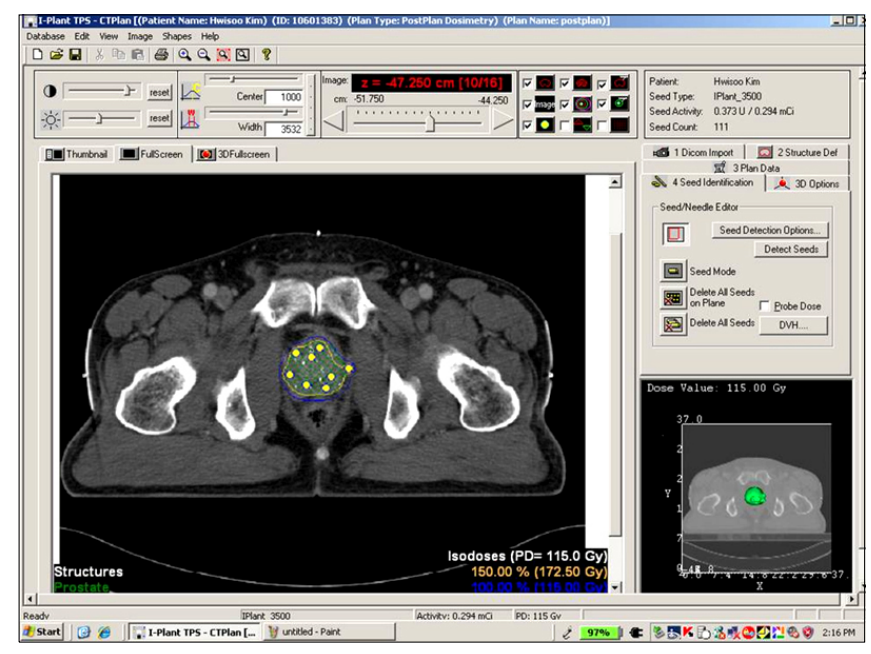

FiG. 3. Postimplant dosimetry by computed tomography scan.

edema to subside, routine evaluation of the implant quality must be performed with computed tomography (CT) scanning (Fig. 3). The average of $V$ and $D$ (percent volume and radiation dose of the prostate exposed to the prescription dose) are calculated. V100 for the prostate (volume of the prostate receiving $100 \%$ of the prescription dose) and D90 of the prostate (dose delivered to $90 \%$ of the prostate) are evaluated. The bladder and rectal dose are also calculated. The American Brachytherapy Society strongly recommends CT-based postimplant prostate dosimetry with quality cutoff points of $\mathrm{V} 100>80 \%$ and $\mathrm{D} 90>90 \%$. Dosimetric implant quality is essential for the optimization of biochemical outcomes [9].

There are two types of seed preparations: stranded and loose. A comparison of loose seeds with stranded seeds re- 
TABLE 2. Biochemical outcomes of 125-I low dose rate brachytherapy according to prognostic risk

\begin{tabular}{lccccc}
\hline \multirow{2}{*}{ Reference series } & Follow-up (mo) & Biochemical & & Recurrence & \multicolumn{2}{c}{ Free rate \% (n) } \\
\cline { 3 - 3 } \cline { 5 - 6 } & & Low risk & & Intermediate risk & High risk \\
\hline Zelefsky et al. [21] & 48 & $88(112)$ & $77(92)$ & $38(22)$ \\
Merrick et al. [22] & 38 & $96(90)$ & $98(121)$ & $79(61)$ \\
Kwok et al. [23] & 94 & $85(41)$ & $63(33)$ & $24(20)$ \\
Kollmeier et al. [24] & 75 & $88(75)$ & $81(70)$ & $65(98)$ \\
Sylvester et al. [25] & 63 & $85(63)$ & $77(92)$ & $45(77)$ \\
\hline
\end{tabular}

ported higher prostate D90 values in the stranded seeds $[10,11]$ because of reduced seed migration. However, other researchers have not observed any dosimetric advantages for stranded seeds [12-15]. These differences may be caused by the operator's technique. The intraoperative prostrate dosimetric parameters were greater for the loose seeds than for the stranded seeds. Within days, however, the dosimetry of the two approaches was similar [15]. Recently, a new one-stage prostate brachytherapy technique (4D Brachytherapy) using a combination of stranded and loose seeds was developed. The use of both stranded and loose seeds may reduce the migration risk of peripherally placed seeds via the venous plexus while maintaining the flexibility to optimize the dose within the prostate and particularly at the apex of the gland [16].

\section{LDR brachytherapy outcomes}

It is difficult to compare results because of the many prognostic variables, including the pretreatment PSA, the Gleason score, the clinical stage, and the implant dose, and because the relapse definitions used vary. The American Society of Therapeutic Radiation Oncology defines PSA failure as three consecutively rising PSA nadir levels. The Phoenix definition is $2.0 \mathrm{ng} / \mathrm{ml}$ plus the nadir point. Patients with a stable but higher PSA level than $>0.5$ $\mathrm{ng} / \mathrm{ml}$ should remain disease free. PSA levels can fluctuate during the follow-up period.

The Seattle group reported that after their initial learning curve was achieved, I-125 brachytherapy improved the long-term outcomes of both low-risk and intermediate-risk patients compared with the patients initially treated at the center. The initial 7-year PSA relapse-free survival results were $70 \%$ and $37 \%$ in the low-risk group and the intermediate-risk group, respectively. After optimizing the implantation technique, the results dramatically improved; the 7-year PSA relapse-free survival rates in the low- and intermediate-risk groups were $87 \%$ and $80 \%$, respectively [17].

Mount Sinai reported the 8-year outcomes of I-125 brachytherapy for 243 patients with a minimum follow-up of 5 years. The 8-year PSA relapse-free survival outcomes for patients with low-risk, intermediate-risk, and high-risk disease were $88 \%, 81 \%$, and $65 \%$, respectively [18]. A prospective randomized controlled study of radical prostatectomy and brachytherapy showed the same biochemical-dis-
TABLE 3. Distribution of patients treated with different treatment methods in meta-analysis [26]

\begin{tabular}{lccc}
\hline Treatment method & Low risk & $\begin{array}{c}\text { Intermediate } \\
\text { risk }\end{array}$ & High risk \\
\hline RP, RARP & 7,183 & 4,175 & 5,349 \\
Brachytherapy & 8,859 & 7,362 & 3,159 \\
Brachytherapy+ADT & - & 165 & 1,231 \\
Protons & 388 & 162 & - \\
EBRT & 4,735 & 2,969 & 3,828 \\
HIFU & 227 & - & - \\
Cryotherapy & - & 175 & 357 \\
\hline
\end{tabular}

Values are presented as no. of patients.

$\mathrm{RP}$, radical prostatectomy; RARP, robot-assisted radical prostatectomy; ADT, androgen deprivation therapy; EBRT, external beam radiotherapy; HIFU, high-intensity focused ultrasound.

ease-free survival rate at 72 months [19] and more long-term excellent outcomes were reported for brachytherapy compared with radical prostatectomy in low-risk or intermediate-risk prostate cancer patients [20]. In this report, the 5-year biochemical recurrence rates for the low-risk and intermediate-risk groups were $96.1 \%$ and $90.6 \%$, respectively, which were not significantly different from radical prostatectomy.

Table 2 summarizes the published biochemical outcomes after LDR brachytherapy according to prognostic risk groups [21-25].

The comparison of the treatment results of the reported studies of radical prostatectomy (open and robot), EBRT (conformal, intensity modulated, and protons), brachytherapy, cryotherapy, and high-intensity focused ultrasound remains difficult to analyze because of reporting inconsistencies and the use of different disease outcome endpoints. The Prostate Cancer Results Study Group performed a comprehensive literature review of more than 18,000 articles (Table 3) [26]. In terms of biochemical-recurrence-free rates, brachytherapy provides excellent outcomes in patients with low-risk disease. For intermediate-risk disease, the combination of EBRT and brachytherapy appears to be equivalent to brachytherapy alone. For high-risk patients, the combination therapies of EBRT and brachytherapy plus or minus androgen deprivation therapy appears superior to more localized treatment methods [26]. 


\section{Acute and late side effects of LDR brachytherapy}

Radiation-induced urethritis and prostatitis result in urinary morbidity, including urinary frequency, urgency, nocturia, hesitancy, weak stream, and terminal dysuria. Lower urinary tract symptoms vary in severity. Various implantation techniques, seed activity, and source distribution patterns have contributed to the wide range of morbidities.

Lower urinary tract symptoms (LUTS) after prostate brachytherapy often develop in 2 to 3 weeks and peak approximately 2 months after the procedure before gradually declining over 6 to 9 months. Approximately $90 \%$ of patients experience grade 1 to 2 acute LUTS during the first 12 months after the procedure [27]. Eight percent of patients experience grade 3 LUTS toxicity and $1.5 \%$ suffer grade 4 toxicity. Late urinary toxicity persists for more than 1 year after brachytherapy in approximately $40 \%$ of patients [8] and ultimately disappears in time. Alpha-blockers and phosphodiesterase-5 (PDE-5) inhibitors may ameliorate LUTS during this early postoperative period. Acute urinary retention requiring catheterization developed in $1 \%$ of the treatment population in our series, whereas other groups have reported incidences of 5.5 to $42 \%$ [28-30].

Urethral stricture and grade 3 late urinary toxicity develop in 9 to $10 \%$ of patients [21]. Grade 4 late urinary toxicity is observed in $0.4 \%$ of patients requiring urinary diversion and a colostomy [21]. Because the severity of urinary toxicity varies according to the exposure level of the urethral radiation, a modified implantation technique that peripherally loads the implant to reduce the urethral radiation dose has been attempted and has resulted in optimal outcomes.

Rectal toxicity after prostate brachytherapy develops in a delayed fashion approximately 1 year after the procedure and persists for 1 to 2 years. Grade 2 and 3 or grade 4 rectal toxicity occurs in 2 to $12 \%$ and 0.4 to $2 \%$ of patients, respectively [31].

The incidence of impotence at 2 years after implantation is $21 \%$, and this rate increases to $42 \%$ at 5 years after the procedure without medication [5]. The addition of neoadjuvant androgen deprivation was reported to have a significant adverse impact on potency after prostate brachytherapy in one study [32], which must be verified.

The posttreatment quality of life secondary to urinary symptoms, the maintenance of sexual function, and the maintenance of normal bowel function are becoming increasingly important to both physicians and patients when deciding which treatment modality is the most suitable.

In a report on long-term toxicity after LDR brachytherapy [33], patient quality of life and sexual function up to 10 years after prostate brachytherapy were analyzed. The results confirmed that brachytherapy had a favorable side effect profile over the long term with regard to potency and urinary and bowel toxicity.

A significant increase in the mean International Prostate Symptom Score at follow-up was observed. A smaller proportion of patients had mild urinary symptoms at follow-up compared with baseline, and a small percentage of men reported severe urinary symptoms at follow-up. The subjects were evaluated for their preoperative erectile function with potency 5 to 10 years later; $62.9 \%$ of patients who had been potent before the operation retained their potency. The use of a PDE-5 inhibitor could improve potency outcomes for men post-brachytherapy, and the men treated were routinely prescribed a PDE-5 inhibitor.

\section{LDR brachytherapy for high-risk prostate cancer}

LDR brachytherapy alone may not deliver a high enough dosage to the periprostatic tissue in patients at high risk for prostate cancer. EBRT should be incorporated with LDR brachytherapy to deliver escalated radiation doses to this risk group. EBRT doses of 45 to 50 Gy are delivered to the prostate and peri-prostatic tissues, and the LDR prescription dose is 90 Gy for Pd-103 implants and 110 Gy for I-125 implants. The interval of the two treatments may be 3 to 4 weeks, and the order is not strict; however, LDR brachytherapy may preferably occur first.

\section{HDR BRACHYTHERAPY}

After the needles are placed within and around the prostate by use of TRUS (Fig. 4A), CT imaging for the treatment planning process is obtained. The urethra and the rectum are identified and a dose plan is created.

HDR temporary prostate brachytherapy using afterloading machines, which contain an Ir-192 source (Fig. 4B), can be applied with EBRT to patients with high-risk prostate cancer. The Ir-192 source moves within the needles for
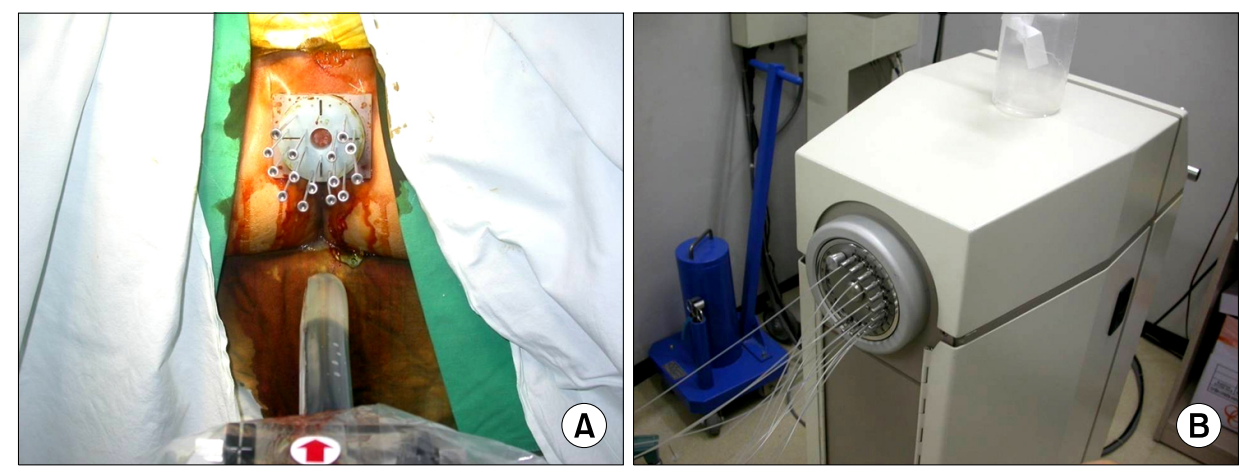

FiG. 4. High dose rate temporary prostate brachytherapy (A) and Ir-192 source (B). 
a short period of time to a number of locations, thus allowing dose distribution to any prostate shape. The implant dose is 12-20 Gy in two to four fractions. Most HDR brachytherapy is combined with EBRT because candidates for this treatment method are intermediate-risk or high-risk prostate cancer groups. The 5-year biochemical relapse-free survival rate for this combination treatment is reported to range from 53 to $84 \%$ [34,35].

HDR brachytherapy can be used as a boost therapy of EBRT [36]. In a phase III randomized trial, EBRT followed by HDR brachytherapy was reported to result in significant improvement in relapse-free survival compared with EBRT alone with a marked reduction in the risk of recurrence and similar incidence of severe late urinary and rectal morbidity. This result is interpreted as effective in achieving dose escalation in the radical radiotherapy of intermediate and poor risk localized prostate cancer [36]. The clear dose response supports not only the role of escalated radiation and the use of hypo-fractionated regimens but also the concept of a much lower $\alpha \beta$ ratio for prostate cancer than for normal genitourinary and rectal epithelia [37]. Other than Ir-192, two different available radionuclide sources, ytterbium-169 and thulium-170, have been tried in HDR brachytherapy [38].

The acute and late morbidity following HDR brachytherapy are similar to those for LDR brachytherapy.

\section{FOCAL BRACHYTHERAPY}

With improved imaging techniques, it is possible to identify men with low- to intermediate-risk prostate cancer for the focal application of brachytherapy. Technically, focal brachytherapy requires advanced imaging using magnetic resonance imaging (MRI) and ultrasonography for localization as well as detailed biopsy results. Although many new ultrasound imaging techniques have been developed, such as B-mode ultrasound, color Doppler ultrasound, contrast enhanced ultrasound, elastography, and sonohistology, there are many limitations in cancer detection focus. New multiparametric MRI techniques, such as diffusion-weighting, dynamic contrast enhancement, and MR spectroscopy are also limited in defining the cancer area. Therefore, advancements in the imaging field are required to apply focal therapy. With this limitation of imaging technique, a mapping biopsy of the prostate should be performed before performing focal therapy.

Among the different permanent seed isotopes available, it was noted that I-125 had the most favorable characteristics considering half-life, tissue edema, and geographic misses. Loose seeds are more favorable than stranded seeds in focal brachytherapy [39].

A number of scenarios in focal brachytherapy can be considered. An ultra-focal or hemi-gland treatment protocol could be considered delivering $145 \mathrm{~Gy}$. In unilateral disease, an index lesion with other clinically insignificant lesions in the contralateral lobe might be treated with 145 Gy given to the side of the index lesion combined with a low- er dose applied to the contralateral side [39].

\section{CONCLUSIONS}

Advancements in imaging technology and the development of new ideal radioisotopes could transform brachytherapy into an ideal therapeutic option for prostate cancer. Emerging new concepts for prostate cancer treatment can also be applied to the small portion of patients in the field of brachytherapy. This trend could change the brachytherapy planning system from an even distribution on the whole gland to an irregular distribution concentrating on lesions.

\section{CONFLICTS OF INTEREST}

The authors have nothing to disclose.

\section{REFERENCES}

1. Whitmore WF Jr, Hilaris B, Grabstald H. Retropubic implantation to iodine 125 in the treatment of prostatic cancer. $J$ Urol 1972;108:918-20.

2. Stamey TA, Caldwell M, McNeal JE, Nolley R, Hemenez M, Downs J. The prostate specific antigen era in the United States is over for prostate cancer: what happened in the last 20 years? J Urol 2004;172(4 Pt 1):1297-301.

3. Park DS, Jang WK, Oh JJ, Jee SH, Shin HS. Application of low dose rate brachytherapy in locally advanced prostate cancer. Korean J Urol Oncol 2008;6:127-32.

4. Stock RG, Stone NN, Wesson MF, DeWyngaert JK. A modified technique allowing interactive ultrasound-guided three-dimensional transperineal prostate implantation. Int J Radiat Oncol Biol Phys 1995;32:219-25.

5. Park DS, Oh JJ, Jang WK, Jee SH, Shin HS. Low-dose-rate brachytherapy for low- and intermediate-risk groups of localized prostate cancer. Korean J Urol 2009;50:656-62.

6. Zelefsky MJ, Yamada Y, Cohen G, Venkatraman ES, Fung AY, Furhang E, et al. Postimplantation dosimetric analysis of permanent transperineal prostate implantation: improved dose distributions with an intraoperative computer-optimized conformal planning technique. Int J Radiat Oncol Biol Phys 2000;48:601-8.

7. Yu Y, Anderson LL, Li Z, Mellenberg DE, Nath R, Schell MC, et al. Permanent prostate seed implant brachytherapy: report of the American Association of Physicists in Medicine Task Group No. 64. Med Phys 1999;26:2054-76.

8. Salembier C, Lavagnini P, Nickers P, Mangili P, Rijnders A, Polo $\mathrm{A}$, et al. Tumour and target volumes in permanent prostate brachytherapy: a supplement to the ESTRO/EAU/EORTC recommendations on prostate brachytherapy. Radiother Oncol 2007; 83:3-10.

9. Merrick GS, Grimm PD, Sylvester J, Blasko JC, Butler WM, Allen ZA, et al. Initial analysis of Pro-Qura: a multi-institutional database of prostate brachytherapy dosimetry. Brachytherapy 2007;6:9-15.

10. Lee WR, deGuzman AF, Tomlinson SK, McCullough DL. Radioactive sources embedded in suture are associated with improved postimplant dosimetry in men treated with prostate brachytherapy. Radiother Oncol 2002;65:123-7.

11. Fagundes HM, Keys RJ, Wojcik MF, Radden MA, Bertelsman CG, Cavanagh WA. Transperineal TRUS-guided prostate brachy- 
therapy using loose seeds versus RAPIDStrand: a dosimetric analysis. Brachytherapy 2004;3:136-40.

12. Fuller DB, Koziol JA, Feng AC. Prostate brachytherapy seed migration and dosimetry: analysis of stranded sources and other potential predictive factors. Brachytherapy 2004;3:10-9.

13. Heysek RV, Gwede CK, Torres-Roca J, Cantor A, Kelley S, Saini AS, et al. A dosimetric analysis of unstranded seeds versus customized stranded seeds in transperineal interstitial permanent prostate seed brachytherapy. Brachytherapy 2006;5:244-50.

14. Reed DR, Wallner KE, Merrick GS, Arthurs S, Mueller A, Cavanagh $\mathrm{W}$, et al. A prospective randomized comparison of stranded vs. loose $125 \mathrm{I}$ seeds for prostate brachytherapy. Brachytherapy 2007;6:129-34.

15. Saibishkumar EP, Borg J, Yeung I, Cummins-Holder C, Landon A, Crook J. Sequential comparison of seed loss and prostate dosimetry of stranded seeds with loose seeds in $125 \mathrm{I}$ permanent implant for low-risk prostate cancer. Int J Radiat Oncol Biol Phys 2009;73:61-8.

16. Langley SE, Laing RW. 4D Brachytherapy, a novel real-time prostate brachytherapy technique using stranded and loose seeds. BJU Int 2012;109 Suppl 1:1-6.

17. Grimm PD, Blasko JC, Sylvester JE, Meier RM, Cavanagh W. 10-year biochemical (prostate-specific antigen) control of prostate cancer with (125)I brachytherapy. Int J Radiat Oncol Biol Phys 2001;51:31-40.

18. Kollmeier MA, Stock RG, Stone N. Biochemical outcomes after prostate brachytherapy with 5-year minimal follow-up: importance of patient selection and implant quality. Int J Radiat Oncol Biol Phys 2003;57:645-53.

19. Giberti C, Chiono L, Gallo F, Schenone M, Gastaldi E. Radical retropubic prostatectomy versus brachytherapy for low-risk prostatic cancer: a prospective study. World J Urol 2009;27:607-12.

20. Fisher CM, Troncoso P, Swanson DA, Munsell MF, Kuban DA, Lee AK, et al. Knife or needles? A cohort analysis of outcomes after radical prostatectomy or brachytherapy for men with low- or intermediate-risk adenocarcinoma of the prostate. Brachytherapy 2012;11:429-34.

21. Zelefsky MJ, Hollister T, Raben A, Matthews S, Wallner KE. Five-year biochemical outcome and toxicity with transperineal CT-planned permanent I-125 prostate implantation for patients with localized prostate cancer. Int J Radiat Oncol Biol Phys 2000;47:1261-6.

22. Merrick GS, Butler WM, Galbreath RW, Lief JH. Five-year biochemical outcome following permanent interstitial brachytherapy for clinical T1-T3 prostate cancer. Int J Radiat Oncol Biol Phys 2001;51:41-8

23. Kwok Y, DiBiase SJ, Amin PP, Naslund M, Sklar G, Jacobs SC. Risk group stratification in patients undergoing permanent (125)I prostate brachytherapy as monotherapy. Int J Radiat Oncol Biol Phys 2002;53:588-94.

24. Kollmeier MA, Pei X, Algur E, Yamada Y, Cox BW, Cohen GN, et al. A comparison of the impact of isotope ((125)I vs. (103)Pd) on toxicity and biochemical outcome after interstitial brachytherapy and external beam radiation therapy for clinically localized prostate cancer. Brachytherapy 2012;11:271-6.

25. Sylvester JE, Blasko JC, Grimm PD, Meier R, Malmgren JA. Ten-year biochemical relapse-free survival after external beam radiation and brachytherapy for localized prostate cancer: the Seattle experience. Int J Radiat Oncol Biol Phys 2003;57:944-52.

26. Grimm P, Billiet I, Bostwick D, Dicker AP, Frank S, Immerzeel $\mathrm{J}$, et al. Comparative analysis of prostate-specific antigen free survival outcomes for patients with low, intermediate and high risk prostate cancer treatment by radical therapy. Results from the Prostate Cancer Results Study Group. BJU Int 2012;109 Suppl 1:22-9.

27. Grimm PD, Blasko JC, Ragde H, Sylvester J, Clarke D. Does brachytherapy have a role in the treatment of prostate cancer? Hematol Oncol Clin North Am 1996;10:653-73.

28. Locke J, Ellis W, Wallner K, Cavanagh W, Blasko J. Risk factors for acute urinary retention requiring temporary intermittent catheterization after prostate brachytherapy: a prospective study. Int J Radiat Oncol Biol Phys 2002;52:712-9.

29. Crook J, McLean M, Catton C, Yeung I, Tsihlias J, Pintilie M. Factors influencing risk of acute urinary retention after TRUS-guided permanent prostate seed implantation. Int J Radiat Oncol Biol Phys 2002;52:453-60.

30. Terk MD, Stock RG, Stone NN. Identification of patients at increased risk for prolonged urinary retention following radioactive seed implantation of the prostate. J Urol 1998;160:1379-82.

31. Emara AM, Chadwick E, Noves JP, Abdelbaky AM, Laing RW, Langley SE. Long-term toxicity and quality of life up to 10 years after low-dose rate brachytherapy for prostate cancer. BJU Int 2012;109:994-1000.

32. Potters L, Torre T, Fearn PA, Leibel SA, Kattan MW. Potency after permanent prostate brachytherapy for localized prostate cancer. Int J Radiat Oncol Biol Phys 2001;50:1235-42.

33. Emara AM, Chadwick E, Nobes JP, Abdelbaky AM, Laing RW, Langley SE. Long-term toxicity and quality of life up to 10 years after low-dose rate brachytherapy for prostate cancer. BJU Int 2012;109:994-1000.

34. Mate TP, Gottesman JE, Hatton J, Gribble M, Van Hollebeke L. High dose-rate afterloading 192Iridium prostate brachytherapy: feasibility report. Int J Radiat Oncol Biol Phys 1998;41:525-33.

35. Dinges S, Deger S, Koswig S, Boehmer D, Schnorr D, Wiegel T, et al. High-dose rate interstitial with external beam irradiation for localized prostate cancer--results of a prospective trial. Radiother Oncol 1998;48:197-202.

36. Hoskin PJ, Rojas AM, Bownes PJ, Lowe GJ, Ostler PJ, Bryant L. Randomised trial of external beam radiotherapy alone or combined with high-dose-rate brachytherapy boost for localised prostate cancer. Radiother Oncol 2012;103:217-22.

37. Martinez AA, Gonzalez J, Ye H, Ghilezan M, Shetty S, Kernen $\mathrm{K}$, et al. Dose escalation improves cancer-related events at 10 years for intermediate- and high-risk prostate cancer patients treated with hypofractionated high-dose-rate boost and external beam radiotherapy. Int J Radiat Oncol Biol Phys 2011;79:363-70.

38. Krishnamurthy D, Weinberg V, Cunha JA, Hsu IC, Pouliot J. Comparison of high-dose rate prostate brachytherapy dose distributions with iridium-192, ytterbium-169, and thulium-170 sources. Brachytherapy 2011;10:461-5.

39. Langley S, Ahmed HU, Al-Qaisieh B, Bostwick D, Dickinson L, Veiga FG, et al. Report of a consensus meeting on focal low dose rate brachytherapy for prostate cancer. BJU Int 2012;109 Suppl 1:7-16. 